\title{
Robust Multivariate Location Estimation in the Existence of Casewise and Cellwise Outliers
}

\author{
Yik-Siong Pang*, Nor Aishah Ahad, Sharipah Soaad Syed Yahaya \\ Department of Mathematics \& Statistics, School of Quantitative Sciences, Universiti Utara Malaysia, 06010 UUM Sintok, Kedah, \\ Malaysia
}

Received November 18, 2020; Revised June 16, 2021; Accepted July 5, 2021

\section{Cite This Paper in the following Citation Styles}

(a): [1] Yik-Siong Pang, Nor Aishah Ahad, Sharipah Soaad Syed Yahaya, "Robust Multivariate Location Estimation in the Existence of Casewise and Cellwise Outliers," Mathematics and Statistics, Vol. 9, No. 5, pp. 653 - 663, 2021. DOI: 10.13189/ms.2021.090505.

(b): Yik-Siong Pang, Nor Aishah Ahad, Sharipah Soaad Syed Yahaya (2021). Robust Multivariate Location Estimation in the Existence of Casewise and Cellwise Outliers. Mathematics and Statistics, 9(5), 653 - 663. DOI: 10.13189/ms.2021.090505.

Copyright $\bigcirc 2021$ by authors, all rights reserved. Authors agree that this article remains permanently open access under the terms of the Creative Commons Attribution License 4.0 International License

\begin{abstract}
Multivariate outliers can exist in two forms, casewise and cellwise. Data collection typically contains unknown proportion and types of outliers which can jeopardize the location estimation and affect research findings. In cases where the two coexist in the same data set, traditional distance-based trimmed mean and coordinate-wise trimmed mean are unable to perform well in estimating location measurement. Distance-based trimmed mean suffers from leftover cellwise outliers after the trimming whereas coordinate-wise trimmed mean is affected by extra casewise outliers. Thus, this paper proposes new robust multivariate location estimation known as $\alpha$-distance-based trimmed median ( $\left.\widehat{\mathbf{M}}_{(\mathbf{M S D}, \boldsymbol{\alpha})}\right)$ to deal with both types of outliers simultaneously in a data set. Simulated data were used to illustrate the feasibility of the new procedure by comparing with the classical mean, classical median and $\alpha$-distance-based trimmed mean. Undeniably, the classical mean performed the best when dealing with clean data, but contrarily on contaminated data. Meanwhile, classical median outperformed distance-based trimmed mean when dealing with both casewise and cellwise outliers, but still affected by the combined outliers' effect. Based on the simulation results, the proposed $\widehat{\mathbf{M}}_{(\mathbf{M S D}, \boldsymbol{\alpha})}$ yields better location estimation on contaminated data compared to the other three estimators considered in this paper. Thus, the proposed $\widehat{\mathbf{M}}_{(\mathbf{M S D}, \boldsymbol{\alpha})}$ can mitigate the issues of outliers and provide a better location estimation.
\end{abstract}

Keywords Multivariate Outliers, Robust Location
Estimation, Distance-Based Trimming, Trimmed Mean, Trimmed Median

\section{Introduction}

\subsection{Multivariate Outliers}

Most parametric statistical tools were derived using the population parameters mean $(\boldsymbol{\mu})$ and (co)variance $(\boldsymbol{\Sigma})$, but most of the time, these population parameters are unknown. Consequently, these parameters are usually estimated from the sample mean and sample covariance, denoted as $\overline{\mathbf{X}}$ and $\mathbf{S}$ respectively. However, Hampel [1] mentioned that real data typically comprises of abnormal observations (outliers). The classical mean with $0 \%$ breakdown point is highly sensitive to outliers, even with only one outlier could divert the estimation from the supposed location and lead to the defect of the least-square-based (co)variance [2-6]. This situation is even complicated in the context of multivariate data as multivariate outliers are harder to be identified.

In multivariate context, outliers occur in a single form or can be a combination of two abnormalities within a single set of data. These outliers can be categorized as either casewise outlier, which refers to one whole observation as a contaminant to the data itself such that the observation originates from another distribution, or cellwise outlier 
which refers to extreme values exist within each variable of the dataset independently similar to those in univariate context [7-8]. Figure 1 illustrates a condition where both casewise and cellwise outliers coexist in a dataset.

Based on Figure 1 , the $z_{9}$ and $z_{10}$ are contaminants originated from another distribution but mistakenly included into Z-distribution. Such contaminations can happen due to sampling error or model misspecification [7], while the extreme values within the components can be some random errors. Not until recent decade, researchers began to realize the situation where both casewise and cellwise outliers present simultaneously. Nevertheless, the existing methods particularly dealt with either casewise or cellwise outliers but not both [9,10]. Thus, the main goal of this article is to introduce a more accurate location estimation method even in the presence of either one or both types of outliers.

\subsection{Robust Approach - Trimming}

Tukey [11] acknowledged the need of a better location estimator dealing with non-normality for Student's $t$-test and suggested the notion of trimming off samples. Trimming refers to a process of removing abnormal observations that caused a sample to be non-normally distributed. In univariate context, the most common trimming procedure for a size $n$ sample is to decide a trimming proportion $(\alpha \%)$ and removes $n \alpha \%$ observations from both end of the ordered statistics.

Generally, multivariate trimming can be done differently via coordinate-wise or distance-based approach. Coordinate-wise trimming (CT) is simple where it treats each of the components of the sample matrix as univariate vector and applies the common trimming procedures. CT is able to retain valid inputs while the outlying inputs were discarded [12]. On the contrary, distance-based approach relies on Mahalanobis Squared Distance (MSD) to determine contaminants for trimming. The common type of distance-based trimming is performed by determining a certain percentile of Chi-Squared distribution to identify multivariate outliers. However, Alloway and Raghavachavari [13] developed a straightforward MSD-based trimmed mean to construct robust Hotelling $T^{2}$ control chart by removing two observations with highest MSD values from the sample. One thing to be noted is that, the trimming procedure introduced by [13] was confined to only 2 observations trimming which can be further extended to $\alpha$-percent distance-based trimmed mean $\left(\overline{\mathbf{X}}_{(\mathbf{M S D}, \boldsymbol{\alpha})}\right)$.

Although the high-distance observations were deemed contaminants and trimmed off, there are possibilities that cellwise outliers may still remain in the multivariate dataset. The remaining data might not be as clean as what we are expecting, for more accurate location estimation. Such situation may cause the application of mean on post-trimming data to be affected by any undetected cellwise outlier. Figure 2 illustrates the post-trimming conditions of a contaminated dataset.

According to Figure 2, the remaining extreme values still exist after employing the distance-based trimming and pose a risk in the estimation of the classical mean $(\overline{\mathbf{Z}})$. In a previous study on Robust Linear Discriminant Analysis [14], the application of median after MSD-based trimming yielded a more consistent performance than of the MSD-based trimmed mean. Therefore, a more reliable location estimator should be applied after the MSD- $\alpha \%$ trimming procedure to alleviate the problem faced when using the highly sensitive classical mean.

$$
\mathbf{Z}=\left[\begin{array}{l}
z_{1} \\
z_{2} \\
z_{3} \\
z_{4} \\
z_{5} \\
z_{6} \\
z_{7} \\
z_{8} \\
z_{9} \\
z_{10}
\end{array}\right]=\left[\begin{array}{cccccc}
0 & 0 & 0 & 0 & \times & 0 \\
0 & 0 & \times & 0 & 0 & 0 \\
0 & 0 & 0 & 0 & 0 & 0 \\
0 & 0 & 0 & \times & 0 & 0 \\
0 & 0 & 0 & 0 & 0 & 0 \\
0 & \times & 0 & 0 & 0 & 0 \\
0 & 0 & 0 & 0 & 0 & 0 \\
\times & 0 & 0 & 0 & 0 & \times \\
\Delta & \Delta & \Delta & \Delta & \Delta & \Delta \\
\Delta & \Delta & \Delta & \Delta & \Delta & \Delta
\end{array}\right]_{10 \times 6} \quad \times \quad \times=\text { extreme value }
$$

Figure 1. Illustration of both casewise and cellwise outliers coexists in a dataset.

$$
\mathbf{Z}_{\text {trimmed }}=\left[\begin{array}{c}
z_{1} \\
z_{2} \\
z_{3} \\
z_{4} \\
z_{5} \\
z_{6} \\
z_{7} \\
z_{8}
\end{array}\right]=\left[\begin{array}{cccccc}
0 & 0 & 0 & 0 & \times & 0 \\
0 & 0 & \times & 0 & 0 & 0 \\
0 & 0 & 0 & 0 & 0 & 0 \\
0 & 0 & 0 & \times & 0 & 0 \\
0 & 0 & 0 & 0 & 0 & 0 \\
0 & \times & 0 & 0 & 0 & 0 \\
0 & 0 & 0 & 0 & 0 & 0 \\
\times & 0 & 0 & 0 & 0 & \times
\end{array}\right]_{8 \times 6} \times=\text { extreme value }
$$

Figure 2. Outliers leftover after Contaminant $x_{9}$ and $x_{10}$ were trimmed using $\alpha$-percent distance-based procedure 


\section{Methodology}

\subsection{Distance-Based Trimmed Mean $\left(\overline{\mathrm{X}}_{(\mathrm{MSD}, \boldsymbol{\alpha})}\right)$}

As mentioned earlier, the trimming process by [13] is rather straight forward. Observations with high MSD measurement will be trimmed off at $\alpha$ percentage. Supposed that $\mathbf{x}_{i j}$ comes from $j$-dimensional feature vectors with $i=1, \ldots, n^{\text {th }}$ sample vector and $j=1, \ldots, d^{\text {th }}$ variable dimension, the classical MSD is obtained via following Eq. 1 [15]:-

$$
\mathbf{M S D}_{\mathrm{i}}=\left(\mathbf{x}_{i j}-\overline{\mathbf{x}}_{j}\right)^{T} \mathbf{S}^{-1}\left(\mathbf{x}_{i j}-\overline{\mathbf{x}}_{j}\right)
$$

where

$\overline{\mathbf{x}}_{\mathrm{j}}=$ classical mean vector of $\mathrm{n}$ samples of $j^{\text {th }}$ dimension

$\mathbf{S}=$ classical covariance matrix of $n$ samples $\times j$ dimension

After MSD vector containing distance measurement for each of the observation was obtained, the following trimming procedure is carried out:-

Step1: Arrange the MSD in ascending order;

Step2: Trim $\alpha \%$ observations with the highest MSD;

Step3: Compute the mean for each dimension based on the remaining observations.

\subsection{Distance-Based Trimmed Median ( $\left.\widehat{\mathrm{M}}_{(\mathrm{MSD}, \alpha)}\right)$}

Directly employing classical median instead of the classical mean on the original data may seem simple and straightforward, but the existence of casewise outliers may distort the estimation of location measure. Therefore, the distance-based trimming procedure extended from Alloway and Raghavachavari [13] is useful in removing casewise outliers. However, for the cases where both casewise and cellwise outliers coexist, the use of classical mean on the remaining post-trimmed data $\left(\mathbf{X}_{\text {trimmed }}\right)$ may still be affected by the remaining cellwise outliers in the dataset. Thus, to overcome this shortcoming of the classical mean, [14] employed median as it is more robust location estimation compared to mean.

To ensure that the outliers are well managed, a two-stage outliers filtration location estimator, known as distance-based trimmed median, is proposed. The first stage is to eliminate casewise outlier by using distance-based approach whereas the use of median estimation acts as a secondary outlier filtration for any possible outlier leftovers (casewise and/or cellwise). The same algorithm presented in Section 2.1. is used to compute the median (instead of the mean in Step 3) for each dimension of the trimmed matrix. A simulation study was conducted to compare the performance of the proposed estimator with other chosen estimators for this study.

\subsection{Trimming Percentage}

Trimming percentages come in various forms. Among the frequently used and suggested percentages of trimming by most researchers are symmetrical trimming of $20 \%$ to $25 \%$ [16], 20\% [17-19], 15\% [20], 10\% to $15 \%$ [21]. For univariate data, the trimming percentage is based on each tail, thus, if the trimming percentage is $10 \%$, the total trimming from left and right tail will be $20 \%$ from each dataset. However, when using MSD- $\alpha \%$ trimming, the total amount of trimming is the amount of the given $n \alpha \%$. Considering trimming percentages from [16-21], the one-tailed distance-based trimming percentage in this study was capped at $40 \%$.

\subsection{Simulation Settings}

The Tukey-Huber Contamination Model (THCM) [22-23] was commonly employed in robust multivariate analyses to generate contaminated data for simulated study. However, the problem with THCM is that the simulation mechanism generates a two-part distribution, i.e., a "clean" part which is normally distributed data whereas the other part consists of rows of contaminated data (casewise outliers). Thus, in order to simulate both casewise and cellwise outliers, THCM was adapted and modified to randomly include cellwise outliers within the simulated data in this study.

Simulated data, matrix $\mathbf{X}_{n \times p}$, with sample size $n$ and variable dimension $p$ were generated according to Eq. 2 around pre-set Location, $L_{\mathrm{p}}$, and simulated according to the settings presented in Table 1.

$$
\begin{aligned}
\mathbf{X}_{n \times \boldsymbol{p}}: & {\left[\left(1-\varepsilon_{1}-\varepsilon_{2}\right) n N_{p}\left(L_{p}, \mathbf{I}_{p}\right)+\varepsilon_{1} n N_{p}\left(L_{p}+\mu_{1}, \mathbf{I}_{p}\right)\right]^{*} } \\
& +\varepsilon_{2} n N_{p}\left(L_{p}+15,25 \mathbf{I}_{p}\right)
\end{aligned}
$$

*where $\varepsilon_{1} n$ were randomly selected from clean data for each dimension $p$ independently to perform $\mu_{1}$ inflation

Table 1. Simulation s Settings

\begin{tabular}{cc}
\hline Contamination Parameters & Controlled Setting \\
\hline Pre-set Location $\left(L_{\mathrm{p}}\right.$ for $\left.\mathrm{p}=1, \ldots, 6\right)$ & $27,10,33,2142,38$ \\
Data Dimensions $(p)$ & 6 \\
Sample Size $(n)$ & 40,100 \\
Cellwise Outliers Proportion $\left(\varepsilon_{1}\right)$ & $0,0.1$ \\
Cellwise Outliers Inflation $\left(\mu_{1}\right)$ & 5,10 \\
Casewise Outliers Proportion $\left(\varepsilon_{2}\right)$ & $0,0.2,0.4$ \\
Trimming Percentage $(\alpha)$ & $0.2,0.3,0.4$ \\
\hline
\end{tabular}

To determine the ability of the estimators in handling outliers, the simulation was conducted according to the following procedure:-

Step 1: Generate data according to the settings in Table 1 ;

Step 2: Compute the location estimators ( $\overline{\mathbf{X}}, \widehat{\mathbf{M}}$, $\overline{\mathbf{X}}_{(\mathbf{M S D}, \boldsymbol{\alpha})}$ and $\left.\widehat{\mathbf{M}}_{(\mathbf{M S D}, \boldsymbol{\alpha})}\right)$;

Step 3: Find the absolute difference of each location estimators and $L_{\mathrm{p}}$;

Step 4: Repeat Step 1 to Step 3 for 10,000 iterations; 
Step 5: Compute the average of each location estimations and absolute difference from the 10,000 iterations.

After obtaining the average absolute difference of estimated location and pre-set location, ANOVA test was conducted to confirm significant difference between the estimators' performance and Tukey's HSD test conducted for post-hoc comparisons between the estimators.

\section{Results}

\subsection{Non-contaminated Data $\left(\varepsilon_{1}=0, \varepsilon_{2}=0\right)$}

Tables 2a and 3a show that classical mean $(\overline{\mathbf{X}})$ is indeed the optimal location estimation with the least average deviation from the pre-set location $\left(L_{\mathrm{p}}\right)$, for each dimension, $p=1,2, \ldots, 6$, for sample sizes of $n=40$ and $n=100$. On the other hand, the performance of classical median $(\widehat{\mathbf{M}})$ is comparable to $\alpha$-distance-based trimmed mean $\left(\overline{\mathbf{X}}_{(\mathbf{M S D}, \boldsymbol{\alpha})}\right)$ with $\alpha=0.2$ or 0.3 at $n=40$ (Table 2a) and $n=100$ (Table 3a). By contrast, the performance of $\alpha$-distance-based trimmed median $\left(\widehat{\mathbf{M}}_{(\mathbf{M S D}, \boldsymbol{\alpha})}\right)$ in the clean data, $n=40$ and $n$ $=100$, do not outperform the other estimators regardless of the trimming percentages $(\alpha=0.2,0.3,0.4)$.

ANOVA test shows significant difference between the estimators' performance when sample size $n=40$ ( $p=$ $0.000)$ and $n=100(p=0.000)$. Tukey's HSD test results were obtained and sorted to rank the estimators based on the difference in $|\mathrm{T}-\mathrm{L}|$ into Tables $2 \mathrm{~b}$ and 3b. Tukey's HSD test confirms the preliminary results in Tables 2a and 3a, with $\overline{\mathbf{X}}$ being the best fit to the pre-set location $L_{\mathrm{p}}$ whereas $\widehat{\mathbf{M}}_{(\mathbf{M S D}, \mathbf{0 . 4})}$ being the least fit to $L_{\mathrm{p}}$.

Table 2a. Simulation Parameter Settings $\left(n=40, \varepsilon_{1}=0, \varepsilon_{2}=0\right)$

\begin{tabular}{|c|c|c|c|c|c|c|c|}
\hline \multirow{2}{*}{\multicolumn{2}{|c|}{ Location Estimators (T) }} & \multicolumn{6}{|c|}{ Pre-set Location $\left(L_{\mathrm{p}}\right)$} \\
\hline & & 27 & 10 & 33 & 21 & 42 & 38 \\
\hline \multirow{2}{*}{$\overline{\mathbf{X}}$} & Average & 27.0004 & 10.0013 & 33.0000 & 21.0015 & 41.9993 & 37.9994 \\
\hline & Ave. Abs. Diff $|\mathrm{T}-\mathrm{L}|$ & 0.1252 & 0.1266 & 0.1265 & 0.1255 & 0.1256 & 0.1264 \\
\hline \multirow{2}{*}{$\widehat{\mathbf{M}}$} & Average & 26.9998 & 10.0019 & 32.9975 & 21.0019 & 42.0004 & 37.9977 \\
\hline & Ave. Abs. Diff $|\mathrm{T}-\mathrm{L}|$ & 0.1548 & 0.1563 & 0.1563 & 0.1543 & 0.1550 & 0.1558 \\
\hline \multirow{2}{*}{$\overline{\mathbf{X}}_{(\mathrm{MSD}, \mathbf{0 . 4})}$} & Average & 27.0004 & 10.0018 & 32.9986 & 20.9997 & 42.0007 & 37.9992 \\
\hline & Ave. Abs. Diff $|\mathrm{T}-\mathrm{L}|$ & 0.1625 & 0.1625 & 0.1652 & 0.1614 & 0.1637 & 0.1629 \\
\hline \multirow{2}{*}{$\overline{\mathbf{X}}_{(\mathbf{M S D}, 0.3)}$} & Average & 26.9996 & 10.0002 & 32.9995 & 21.0008 & 41.9995 & 37.9989 \\
\hline & Ave. Abs. Diff $|\mathrm{T}-\mathrm{L}|$ & 0.1553 & 0.1561 & 0.1570 & 0.1545 & 0.1558 & 0.1558 \\
\hline \multirow{2}{*}{$\overline{\mathbf{X}}_{(\mathrm{MSD}, 0.2)}$} & Average & 27.0006 & 10.0007 & 32.9996 & 21.0012 & 41.9999 & 37.9989 \\
\hline & Ave. Abs. Diff $|\mathrm{T}-\mathrm{L}|$ & 0.1474 & 0.1484 & 0.1482 & 0.1467 & 0.1485 & 0.1469 \\
\hline \multirow{2}{*}{$\widehat{\mathbf{M}}_{(\mathrm{MSD}, \mathbf{0 . 4})}$} & Average & 26.9993 & 10.0023 & 32.9952 & 21.0014 & 42.0008 & 37.9979 \\
\hline & Ave. Abs. Diff $|\mathrm{T}-\mathrm{L}|$ & 0.1927 & 0.1904 & 0.1933 & 0.1911 & 0.1917 & 0.1921 \\
\hline \multirow{2}{*}{$\widehat{\mathbf{M}}_{(\mathrm{MSD}, 0.3)}$} & Average & 26.9985 & 10.0008 & 32.9957 & 21.0019 & 42.0002 & 37.9979 \\
\hline & Ave. Abs. Diff $|\mathrm{T}-\mathrm{L}|$ & 0.1834 & 0.1818 & 0.1855 & 0.1829 & 0.1846 & 0.1829 \\
\hline \multirow{2}{*}{$\widehat{\mathbf{M}}_{(\mathrm{MSD}, 0.2)}$} & Average & 26.9995 & 10.0013 & 32.9970 & 21.0013 & 42.0011 & 37.9978 \\
\hline & Ave. Abs. Diff |T-L $\mid$ & 0.1754 & 0.1747 & 0.1760 & 0.1738 & 0.1770 & 0.1742 \\
\hline
\end{tabular}

Table 2b. Estimators Ranking based on Tukey HSD Test $\left(n=40, \varepsilon_{1}=0, \varepsilon_{2}=0\right)$

\begin{tabular}{llllllll}
\hline Worst Fit & & & & & & \multicolumn{1}{c}{ Best Fit } \\
\hline$\widehat{\mathbf{M}}_{(\mathrm{MSD}, 0.4)}$ & $\widehat{\mathbf{M}}_{(\mathrm{MSD}, 0.3)}$ & $\widehat{\mathbf{M}}_{(\mathrm{MSD}, 0.2)}$ & $\overline{\mathbf{X}}_{(\mathrm{MSD}, 0.4)}$ & $\overline{\mathbf{X}}_{(\mathrm{MSD}, 0.3)}{ }^{*}$ & $\widehat{\mathbf{M}}^{*}$ & $\overline{\mathbf{X}}_{(\mathrm{MSD}, 0.2)}$ & $\overline{\mathbf{X}}$ \\
\hline
\end{tabular}

Note: All pairwise Tukey HSD results are significant at 0.05 significant level except ${ }^{*} \overline{\mathrm{X}}_{(\mathrm{MSD}, 0.3)}$ and $\widehat{\mathrm{M}}$ are insignificant with $p$-value of 0.999 
Table 3a. Simulation Parameter Settings $\left(n=100, \varepsilon_{1}=0, \varepsilon_{2}=0\right)$

\begin{tabular}{|c|c|c|c|c|c|c|c|}
\hline \multirow{2}{*}{\multicolumn{2}{|c|}{ Location Estimators (T) }} & \multicolumn{6}{|c|}{ Pre-set Location $\left(L_{\mathrm{p}}\right)$} \\
\hline & & 27 & 10 & 33 & 21 & 42 & 38 \\
\hline \multirow{2}{*}{$\overline{\mathbf{X}}$} & Average & 26.9990 & 9.9998 & 32.9998 & 21.0000 & 42.0002 & 37.9987 \\
\hline & Ave. Abs. Diff $|\mathrm{T}-\mathrm{L}|$ & 0.0799 & 0.0797 & 0.0797 & 0.0804 & 0.0791 & 0.0790 \\
\hline \multirow{2}{*}{$\widehat{\mathbf{M}}$} & Average & 26.9997 & 9.9998 & 32.9998 & 21.0001 & 41.9991 & 37.9984 \\
\hline & Ave. Abs. Diff $|\mathrm{T}-\mathrm{L}|$ & 0.0994 & 0.1001 & 0.0997 & 0.1000 & 0.0991 & 0.0998 \\
\hline \multirow{2}{*}{$\overline{\mathbf{X}}_{(\mathbf{M S D}, \mathbf{0 . 4})}$} & Average & 26.9986 & 9.9993 & 32.9996 & 21.0014 & 42.0008 & 37.9997 \\
\hline & Ave. Abs. Diff $|\mathrm{T}-\mathrm{L}|$ & 0.1032 & 0.1034 & 0.1023 & 0.1035 & 0.1025 & 0.1035 \\
\hline \multirow{2}{*}{$\overline{\mathbf{X}}_{(\mathrm{MSD}, 0.3)}$} & Average & 26.9986 & 10.0001 & 33.0000 & 21.0011 & 42.0001 & 37.9998 \\
\hline & Ave. Abs. Diff $|\mathrm{T}-\mathrm{L}|$ & 0.0982 & 0.0989 & 0.0975 & 0.0990 & 0.0976 & 0.0989 \\
\hline \multirow{2}{*}{$\overline{\mathbf{X}}_{(\mathrm{MSD}, 0.2)}$} & Average & 26.9985 & 9.9999 & 33.0004 & 21.0006 & 41.9996 & 37.9989 \\
\hline & Ave. Abs. Diff $|\mathrm{T}-\mathrm{L}|$ & 0.0939 & 0.0940 & 0.0930 & 0.0943 & 0.0928 & 0.0936 \\
\hline \multirow{2}{*}{$\widehat{\mathbf{M}}_{(\mathbf{M S D}, 0.4)}$} & Average & 26.9989 & 9.9989 & 32.9990 & 21.0016 & 41.9998 & 37.9990 \\
\hline & Ave. Abs. Diff $|\mathrm{T}-\mathrm{L}|$ & 0.1232 & 0.1242 & 0.1223 & 0.1233 & 0.1225 & 0.1234 \\
\hline \multirow{2}{*}{$\widehat{\mathbf{M}}_{(\mathrm{MSD}, 0.3)}$} & Average & 26.9990 & 10.0004 & 32.9992 & 21.0007 & 41.9993 & 37.9997 \\
\hline & Ave. Abs. Diff $|\mathrm{T}-\mathrm{L}|$ & 0.1170 & 0.1184 & 0.1170 & 0.1178 & 0.1169 & 0.1176 \\
\hline \multirow{2}{*}{$\widehat{\mathbf{M}}_{(\mathrm{MSD}, 0.2)}$} & Average & 26.9994 & 10.0004 & 32.9994 & 21.0004 & 41.9985 & 37.9988 \\
\hline & Ave. Abs. Diff $|\mathrm{T}-\mathrm{L}|$ & 0.1118 & 0.1130 & 0.1122 & 0.1129 & 0.1123 & 0.1118 \\
\hline
\end{tabular}

Table 3b. Estimators Ranking based on Tukey HSD Test $\left(n=100, \varepsilon_{1}=0, \varepsilon_{2}=0\right)$

\begin{tabular}{|c|c|c|c|c|c|c|c|}
\hline Worst Fit & & & & & & & Best Fit \\
\hline$\widehat{\mathbf{M}}_{(\mathbf{M S D}, 0.4)}$ & $\widehat{\mathbf{M}}_{(\mathbf{M S D}, 0.3)}$ & $\widehat{\mathbf{M}}_{(\mathrm{MSD}, 0.2)}$ & $\overline{\mathbf{X}}_{(\mathbf{M S D}, \mathbf{0 . 4})}$ & $\widehat{\mathbf{M}}$ & $\overline{\mathbf{X}}_{(\mathbf{M S D}, \mathbf{0 . 3})}$ & $\overline{\mathbf{X}}_{(\mathbf{M S D}, 0.2)}$ & $\overline{\mathbf{X}}$ \\
\hline
\end{tabular}

Note: All pairwise Tukey HSD results are significant at 0.05 significant level

\subsection{Data Contamination at $\varepsilon_{1}=0.1$ and $\varepsilon_{2}=0.2$}

\subsubsection{Mild Cellwise Outliers $\left(\mu_{1}=5\right)$}

As cellwise outliers and casewise outliers present in the samples, $\overline{\mathbf{X}}$ is inevitably affected. Tables $4 \mathrm{a}$ and 5a show that $\overline{\mathbf{X}}$ deviates the most from $L_{\mathrm{p}}$ compared to other estimators at both sample sizes, $n=40$ and $n=100$. On the other hand, $\overline{\mathbf{X}}_{(\mathbf{M S D}, \boldsymbol{\alpha})}$ significantly outperforms $\overline{\mathbf{X}}$ with better accuracy as trimming percentage $(\alpha)$ increases. Simulation also shows that $\widehat{\mathbf{M}}$ is better than $\overline{\mathbf{X}}$ and $\overline{\mathbf{X}}_{(\mathbf{M S D}, \boldsymbol{\alpha})}$ in handling outliers' influence. Meanwhile, $\widehat{\mathbf{M}}_{(\mathbf{M S D}, \boldsymbol{\alpha})}$ illustrates a better and more stable performance compared to the other estimators.

Similarly, ANOVA test indicates significant difference amongst the estimators' performance when $n=40$ ( $p=$ $0.000)$ and $n=100(p=0.000)$. The rankings according to Tukey's HSD test determine $\widehat{\mathbf{M}}_{(\mathbf{M S D}, \mathbf{0 . 2})}$ as the best fit while $\overline{\mathbf{X}}$ is the least fit location estimation for both sample sizes $n=40$ (Table 4b) and $n=100$ (Table 5b).

\subsubsection{Severe Cellwise Outliers $\left(\mu_{1}=10\right)$}

When the cellwise outliers' inflation increases from 5 to $10, \overline{\mathbf{X}}$ further deteriorates as shown in Tables 6a and 7a. The deviation of $\overline{\mathbf{X}}_{(\mathbf{M S D}, \boldsymbol{\alpha})}$ began to increase following cellwise outliers' inflation increment. Besides that, $\widehat{\mathbf{M}}$ maintains with relatively similar results despite the cellwise outliers' inflation increment. Notably, although cellwise outliers' weight increases, $\widehat{\mathbf{M}}_{(\mathbf{M S D}, \boldsymbol{\alpha})}$ 's deviation from $L_{\mathrm{p}}$ decreases with $\alpha=0.3$ and $\alpha=0.4$, while maintains with $\alpha=0.2$.

ANOVA results with $p=0.000$ are reported, reported for both sample sizes $n=40$ and $n=100$. Tables $6 \mathrm{~b}$ and $7 \mathrm{~b}$ show that $\overline{\mathbf{X}}$ is the least fit estimation for both $n=40$ and $n$ $=100$. On the contrary, $\widehat{\mathbf{M}}_{(\mathbf{M S D}, \boldsymbol{\alpha})}$ did not have significant difference $(p$-value $=1.000)$ for $\alpha=0.2,0.3,0.4$ with sample size $n=40$ (Table 6b). Similarly, Table 7b $(n=100)$ also reported no difference between $\widehat{\mathbf{M}}_{(\mathbf{M S D}, \mathbf{0 . 3})}$ and $\widehat{\mathbf{M}}_{(\mathbf{M S D}, \mathbf{0 . 4})}$. However, the rankings between the three $\widehat{\mathbf{M}}_{(\mathbf{M S D}, \boldsymbol{\alpha})}$ estimations against other estimators are shown in the following tables. 
Table 4a. Simulation Parameter Settings ( $\left.n=40, \varepsilon_{1}=0.1, \mu_{1}=5, \varepsilon_{2}=0.2\right)$

\begin{tabular}{|c|c|c|c|c|c|c|c|}
\hline \multirow{2}{*}{\multicolumn{2}{|c|}{ Location Estimators (T) }} & \multicolumn{6}{|c|}{ Pre-set Location $\left(L_{\mathrm{p}}\right)$} \\
\hline & & \multirow{2}{*}{$\begin{array}{c}\mathbf{2 7} \\
30.5387\end{array}$} & \multirow{2}{*}{$\frac{10}{13.4922}$} & \multirow{2}{*}{$\begin{array}{c}33 \\
36.4967\end{array}$} & \multirow{2}{*}{$\frac{\mathbf{2 1}}{24.4955}$} & \multirow{2}{*}{$\begin{array}{c}42 \\
45.5179\end{array}$} & \multirow{2}{*}{$\begin{array}{c}\mathbf{3 8} \\
41.4825\end{array}$} \\
\hline$\overline{\mathbf{y}}$ & Average & & & & & & \\
\hline $\mathbf{X}$ & Ave. Abs. Diff $|\mathrm{T}-\mathrm{L}|$ & 3.5695 & 3.5273 & 3.5300 & 3.5253 & 3.5512 & 3.5185 \\
\hline \multirow{2}{*}{$\widehat{\mathbf{M}}$} & Average & 27.3451 & 10.3439 & 33.3445 & 21.3451 & 42.3444 & 38.3412 \\
\hline & Ave. Abs. Diff $|\mathrm{T}-\mathrm{L}|$ & 0.3689 & 0.3661 & 0.3672 & 0.3685 & 0.3654 & 0.3645 \\
\hline \multirow{2}{*}{$\overline{\mathbf{X}}_{(\mathrm{MSD}, 0.4)}$} & Average & 27.4618 & 10.4611 & 33.4630 & 21.4618 & 42.4627 & 38.4546 \\
\hline & Ave. Abs. Diff $|\mathrm{T}-\mathrm{L}|$ & 0.4819 & 0.4815 & 0.4825 & 0.4824 & 0.4820 & 0.4737 \\
\hline \multirow{2}{*}{$\overline{\mathbf{X}}_{(\mathrm{MSD}, 0.3)}$} & Average & 27.5377 & 10.5365 & 33.5396 & 21.5369 & 42.5358 & 38.5307 \\
\hline & Ave. Abs. Diff $|\mathrm{T}-\mathrm{L}|$ & 0.5423 & 0.5419 & 0.5444 & 0.5421 & 0.5415 & 0.5355 \\
\hline \multirow{2}{*}{$\overline{\mathbf{X}}_{(\mathrm{MSD}, 0.2)}$} & Average & 27.6289 & 10.6290 & 33.6299 & 21.6305 & 42.6284 & 38.6265 \\
\hline & Ave. Abs. Diff $|\mathrm{T}-\mathrm{L}|$ & 0.6290 & 0.6291 & 0.6301 & 0.6306 & 0.6284 & 0.6265 \\
\hline \multirow{2}{*}{$\widehat{\mathbf{M}}_{(\mathrm{MSD}, \mathbf{0 . 4})}$} & Average & 27.1557 & 10.1588 & 33.1553 & 21.1550 & 42.1567 & 38.1509 \\
\hline & Ave. Abs. Diff $|\mathrm{T}-\mathrm{L}|$ & 0.2537 & 0.2511 & 0.2515 & 0.2530 & 0.2511 & 0.2493 \\
\hline \multirow{2}{*}{$\widehat{\mathbf{M}}_{(\mathbf{M S D}, 0.3)}$} & Average & 27.1659 & 10.1689 & 33.1680 & 21.1645 & 42.1665 & 38.1620 \\
\hline & Ave. Abs. Diff $|\mathrm{T}-\mathrm{L}|$ & 0.2442 & 0.2423 & 0.2441 & 0.2438 & 0.2411 & 0.2398 \\
\hline \multirow{2}{*}{$\widehat{\mathbf{M}}_{(\mathrm{MSD}, 0.2)}$} & Average & 27.1776 & 10.1806 & 33.1795 & 21.1787 & 42.1794 & 38.1766 \\
\hline & Ave. Abs. Diff $|\mathrm{T}-\mathrm{L}|$ & 0.2380 & 0.2376 & 0.2374 & 0.2373 & 0.2360 & 0.2360 \\
\hline
\end{tabular}

Table 4b. Estimators Ranking based on Tukey HSD Test $\left(n=40, \varepsilon_{1}=0.1, \mu_{1}=5, \varepsilon_{2}=0.2\right)$

\begin{tabular}{|c|c|c|c|c|c|c|c|}
\hline Worst Fit & & & & & & & Best Fit \\
\hline$\overline{\mathbf{X}}$ & $\overline{\mathbf{X}}_{(\mathbf{M S D}, 0.2)}$ & $\overline{\mathbf{X}}_{(\mathbf{M S D}, \mathbf{0 . 3 )}}$ & $\overline{\mathbf{X}}_{(\mathbf{M S D}, \mathbf{0 . 4})}$ & $\widehat{\mathbf{M}}$ & $\widehat{\mathbf{M}}_{(\mathbf{M S D}, 0.4)}{ }^{*}$ & $\widehat{\mathbf{M}}_{(\mathrm{MSD}, 0.3)^{*, * *}}$ & $\widehat{\mathbf{M}}_{(\mathbf{M S D}, 0.2)}{ }^{* *}$ \\
\hline
\end{tabular}

Note: All pairwise Tukey HSD results are significant at 0.05 significant level except ${ }^{*} \widehat{M}_{(\mathrm{MSD}, 0.4)}$ and $\widehat{\mathrm{M}}_{(\mathrm{MSD}, 0.3)}$ are insignificant with $p$-value of 0.375 ;

${ }^{* *} \widehat{\mathrm{M}}_{(\mathrm{MSD}, 0.3)}$ and $\widehat{\mathrm{M}}_{(\mathrm{MSD}, 0.2)}$ are insignificant with $p$-value of 0.881 .

Table 5a. Simulation Parameter Settings ( $\left.n=100, \varepsilon_{1}=0.1, \mu_{1}=5, \varepsilon_{2}=0.2\right)$

\begin{tabular}{|c|c|c|c|c|c|c|c|}
\hline \multirow{2}{*}{\multicolumn{2}{|c|}{ Location Estimators (T) }} & \multicolumn{6}{|c|}{ Pre-set Location $\left(L_{\mathrm{p}}\right)$} \\
\hline & & 27 & 10 & 33 & 21 & 42 & 38 \\
\hline \multirow{2}{*}{$\overline{\mathbf{X}}$} & Average & 30.5047 & 13.4883 & 36.4974 & 24.4988 & 45.4872 & 41.4940 \\
\hline & Ave. Abs. Diff $|\mathrm{T}-\mathrm{L}|$ & 3.5052 & 3.4886 & 3.4977 & 3.4992 & 3.4876 & 3.4944 \\
\hline \multirow{2}{*}{$\widehat{\mathbf{M}}$} & Average & 27.3417 & 10.3439 & 33.3443 & 21.3439 & 42.3445 & 38.3432 \\
\hline & Ave. Abs. Diff $|\mathrm{T}-\mathrm{L}|$ & 0.3441 & 0.3463 & 0.3466 & 0.3464 & 0.3468 & 0.3457 \\
\hline \multirow{2}{*}{$\overline{\mathbf{X}}_{(\mathbf{M S D}, 0.4)}$} & Average & 27.4985 & 10.4965 & 33.4931 & 21.4979 & 42.4984 & 38.4964 \\
\hline & Ave. Abs. Diff $|\mathrm{T}-\mathrm{L}|$ & 0.5009 & 0.4990 & 0.4955 & 0.5003 & 0.5011 & 0.4991 \\
\hline \multirow{2}{*}{$\overline{\mathbf{X}}_{(\mathrm{MSD}, 0.3)}$} & Average & 27.5498 & 10.5501 & 33.5485 & 21.5495 & 42.5517 & 38.5509 \\
\hline & Ave. Abs. Diff $|\mathrm{T}-\mathrm{L}|$ & 0.5499 & 0.5504 & 0.5487 & 0.5499 & 0.5520 & 0.5512 \\
\hline \multirow{2}{*}{$\overline{\mathbf{X}}_{(\mathrm{MSD}, 0.2)}$} & Average & 27.6243 & 10.6246 & 33.6242 & 21.6252 & 42.6256 & 38.6252 \\
\hline & Ave. Abs. Diff $|\mathrm{T}-\mathrm{L}|$ & 0.6243 & 0.6246 & 0.6242 & 0.6252 & 0.6256 & 0.6252 \\
\hline \multirow{2}{*}{$\widehat{\mathbf{M}}_{(\mathrm{MSD}, 0.4)}$} & Average & 27.1892 & 10.1864 & 33.1870 & 21.1873 & 42.1897 & 38.1875 \\
\hline & Ave. Abs. Diff $|\mathrm{T}-\mathrm{L}|$ & 0.2178 & 0.2152 & 0.2172 & 0.2167 & 0.2190 & 0.2160 \\
\hline \multirow{2}{*}{$\widehat{\mathbf{M}}_{(\mathbf{M S D}, 0.3)}$} & Average & 27.1822 & 10.1808 & 33.1810 & 21.1803 & 42.1835 & 38.1813 \\
\hline & Ave. Abs. Diff $|\mathrm{T}-\mathrm{L}|$ & 0.2050 & 0.2031 & 0.2042 & 0.2034 & 0.2060 & 0.2044 \\
\hline \multirow{2}{*}{$\widehat{\mathbf{M}}_{(\mathbf{M S D}, 0.2)}$} & Average & 27.1788 & 10.1787 & 33.1792 & 21.1791 & 42.1808 & 38.1789 \\
\hline & Ave. Abs. Diff $|\mathrm{T}-\mathrm{L}|$ & 0.1957 & 0.1957 & 0.1960 & 0.1957 & 0.1970 & 0.1959 \\
\hline
\end{tabular}

Table 5b. Estimators Ranking based on Tukey HSD Test ( $\left.n=100, \varepsilon_{1}=0.1, \mu_{1}=5, \varepsilon_{2}=0.2\right)$

\begin{tabular}{|c|c|c|c|c|c|c|c|}
\hline Worst Fit & & & & & & & Best Fit \\
\hline$\overline{\mathbf{X}}$ & $\overline{\mathbf{X}}_{(\mathrm{MSD}, 0.2)}$ & $\overline{\mathbf{X}}_{(\mathrm{MSD}, 0.3)}$ & $\overline{\mathbf{X}}_{(\mathbf{M S D}, \mathbf{0 . 4})}$ & $\widehat{\mathbf{M}}$ & $\widehat{\mathbf{M}}_{(\mathrm{MSD}, 0.4)}$ & $\widehat{\mathbf{M}}_{(\mathbf{M S D}, 0.3)}$ & $\widehat{\mathbf{M}}_{(\mathrm{MSD}, 0.2)}$ \\
\hline
\end{tabular}

Note: All pairwise Tukey HSD results are significant at 0.05 significant level 
Table 6a. Simulation Parameter Settings ( $\left.n=40, \varepsilon_{1}=0.1, \mu_{1}=10, \varepsilon_{2}=0.2\right)$

\begin{tabular}{|c|c|c|c|c|c|c|c|}
\hline \multirow{2}{*}{\multicolumn{2}{|c|}{ Location Estimators (T) }} & \multicolumn{6}{|c|}{ Pre-set Location $\left(L_{\mathrm{p}}\right)$} \\
\hline & & 27 & 10 & 33 & 21 & 42 & 38 \\
\hline \multirow{2}{*}{$\overline{\mathbf{X}}$} & Average & 31.0387 & 13.9922 & 36.9967 & 24.9955 & 46.0179 & 41.9825 \\
\hline & Ave. Abs. Diff $|\mathrm{T}-\mathrm{L}|$ & 4.0538 & 4.0091 & 4.0116 & 4.0094 & 4.0344 & 3.9999 \\
\hline \multirow{2}{*}{$\widehat{\mathbf{M}}$} & Average & 27.3452 & 10.3440 & 33.3457 & 21.3443 & 42.3419 & 38.3420 \\
\hline & Ave. Abs. Diff $|\mathrm{T}-\mathrm{L}|$ & 0.3694 & 0.3660 & 0.3679 & 0.3691 & 0.3638 & 0.3653 \\
\hline \multirow{2}{*}{$\overline{\mathbf{X}}_{(\mathbf{M S D}, \mathbf{0 . 4})}$} & Average & 27.8204 & 10.8152 & 33.8243 & 21.8228 & 42.8218 & 38.8073 \\
\hline & Ave. Abs. Diff $|\mathrm{T}-\mathrm{L}|$ & 0.8408 & 0.8358 & 0.8454 & 0.8437 & 0.8420 & 0.8276 \\
\hline \multirow{2}{*}{$\overline{\mathbf{X}}_{(\mathbf{M S D}, \mathbf{0 . 3})}$} & Average & 28.0346 & 11.0294 & 34.0383 & 22.0397 & 43.0352 & 39.0264 \\
\hline & Ave. Abs. Diff $|\mathrm{T}-\mathrm{L}|$ & 1.0379 & 1.0330 & 1.0412 & 1.0429 & 1.0380 & 1.0293 \\
\hline \multirow{2}{*}{$\overline{\mathbf{X}}_{(\mathbf{M S D}, 0.2)}$} & Average & 28.2664 & 11.2636 & 34.2650 & 22.2639 & 43.2642 & 39.2592 \\
\hline & Ave. Abs. Diff $|\mathrm{T}-\mathrm{L}|$ & 1.2664 & 1.2636 & 1.2650 & 1.2639 & 1.2643 & 1.2592 \\
\hline \multirow{2}{*}{$\widehat{\mathbf{M}}_{(\mathrm{MSD}, 0.4)}$} & Average & 27.1296 & 10.1314 & 33.1344 & 21.1326 & 42.1279 & 38.1287 \\
\hline & Ave. Abs. Diff $|\mathrm{T}-\mathrm{L}|$ & 0.2398 & 0.2386 & 0.2414 & 0.2418 & 0.2357 & 0.2391 \\
\hline \multirow{2}{*}{$\widehat{\mathbf{M}}_{(\mathrm{MSD}, 0.3)}$} & Average & 27.1549 & 10.1564 & 33.1587 & 21.1573 & 42.1543 & 38.1544 \\
\hline & Ave. Abs. Diff $|\mathrm{T}-\mathrm{L}|$ & 0.2381 & 0.2361 & 0.2390 & 0.2396 & 0.2341 & 0.2364 \\
\hline \multirow{2}{*}{$\widehat{\mathbf{M}}_{(\mathrm{MSD}, 0.2)}$} & Average & 27.1789 & 10.1808 & 33.1814 & 21.1790 & 42.1784 & 38.1779 \\
\hline & Ave. Abs. Diff $|\mathrm{T}-\mathrm{L}|$ & 0.2398 & 0.2377 & 0.2393 & 0.2398 & 0.2363 & 0.2367 \\
\hline
\end{tabular}

Table 6b. Estimators Ranking based on Tukey HSD Test ( $\left.n=40, \varepsilon_{1}=0.1, \mu_{1}=10, \varepsilon_{2}=0.2\right)$

\begin{tabular}{|c|c|c|c|c|c|c|c|}
\hline Worst Fit & & & & & & & Best Fit \\
\hline$\overline{\mathbf{X}}$ & $\overline{\mathbf{X}}_{(\mathbf{M S D}, 0.2)}$ & $\overline{\mathbf{X}}_{(\mathrm{MSD}, 0.3)}$ & $\overline{\mathbf{X}}_{(\mathbf{M S D}, 0.4)}$ & $\widehat{\mathbf{M}}$ & $\widehat{\mathbf{M}}_{(\mathbf{M S D}, 0.4)}{ }^{*}$ & $\widehat{\mathbf{M}}_{(\mathrm{MSD}, 0.2)}{ }^{*, * * *}$ & $\widehat{\mathbf{M}}_{(\mathrm{MSD}, 0.3)}{ }^{* *}$ \\
\hline
\end{tabular}

Note: All pairwise Tukey HSD results are significant at 0.05 significant level except ${ }^{*} \widehat{\mathrm{M}}_{(\mathrm{MSD}, 0.4)}$ and $\widehat{\mathrm{M}}_{(\mathrm{MSD}, 0.2)}$ are insignificant with $p$-value of 1.000 ;

${ }^{* *} \widehat{\mathrm{M}}_{(\mathrm{MSD}, 0.2)}$ and $\widehat{\mathrm{M}}_{(\mathrm{MSD}, 0.3)}$ are insignificant with $p$-value of 1.000 .

Table 7a. Simulation Parameter Settings ( $\left.n=100, \varepsilon_{1}=0.1, \mu_{1}=10, \varepsilon_{2}=0.2\right)$

\begin{tabular}{|c|c|c|c|c|c|c|c|}
\hline \multirow{2}{*}{\multicolumn{2}{|c|}{ Location Estimators (T) }} & \multicolumn{6}{|c|}{ Pre-set Location $\left(L_{\mathrm{p}}\right)$} \\
\hline & & 27 & 10 & 33 & 21 & 42 & 38 \\
\hline \multirow{2}{*}{$\overline{\mathbf{X}}$} & Average & 31.0047 & 13.9883 & 36.9974 & 24.9988 & 45.9872 & 41.9940 \\
\hline & Ave. Abs. Diff $|\mathrm{T}-\mathrm{L}|$ & 4.0049 & 3.9883 & 3.9974 & 3.9988 & 3.9872 & 3.9940 \\
\hline \multirow{2}{*}{$\widehat{\mathbf{M}}$} & Average & 27.3415 & 10.3438 & 33.3442 & 21.3430 & 42.3446 & 38.3428 \\
\hline & Ave. Abs. Diff $|\mathrm{T}-\mathrm{L}|$ & 0.3440 & 0.3464 & 0.3466 & 0.3456 & 0.3471 & 0.3453 \\
\hline \multirow{2}{*}{$\overline{\mathbf{X}}_{(\mathrm{MSD}, 0.4)}$} & Average & 27.7964 & 10.7883 & 33.7880 & 21.7918 & 42.7905 & 38.7934 \\
\hline & Ave. Abs. Diff $|\mathrm{T}-\mathrm{L}|$ & 0.8004 & 0.7926 & 0.7919 & 0.7955 & 0.7944 & 0.7973 \\
\hline \multirow{2}{*}{$\overline{\mathbf{X}}_{(\mathrm{MSD}, 0.3)}$} & Average & 28.0090 & 11.0062 & 34.0055 & 22.0073 & 43.0058 & 39.0107 \\
\hline & Ave. Abs. Diff $|\mathrm{T}-\mathrm{L}|$ & 1.0092 & 1.0064 & 1.0055 & 1.0075 & 1.0058 & 1.0109 \\
\hline \multirow{2}{*}{$\overline{\mathbf{X}}_{(\mathrm{MSD}, 0.2)}$} & Average & 28.2506 & 11.2505 & 34.2503 & 22.2512 & 43.2519 & 39.2512 \\
\hline & Ave. Abs. Diff |T-L $\mid$ & 1.2506 & 1.2505 & 1.2503 & 1.2512 & 1.2519 & 1.2512 \\
\hline \multirow{2}{*}{$\widehat{\mathbf{M}}_{(\mathbf{M S D}, \mathbf{0 . 4})}$} & Average & 27.1368 & 10.1367 & 33.1384 & 21.1371 & 42.1394 & 38.1378 \\
\hline & Ave. Abs. Diff $|\mathrm{T}-\mathrm{L}|$ & 0.1815 & 0.1804 & 0.1830 & 0.1816 & 0.1843 & 0.1809 \\
\hline \multirow{2}{*}{$\widehat{\mathbf{M}}_{(\mathrm{MSD}, 0.3)}$} & Average & 27.1570 & 10.1574 & 33.1590 & 21.1570 & 42.1582 & 38.1584 \\
\hline & Ave. Abs. Diff $|\mathrm{T}-\mathrm{L}|$ & 0.1861 & 0.1865 & 0.1875 & 0.1863 & 0.1875 & 0.1866 \\
\hline \multirow{2}{*}{$\widehat{\mathbf{M}}_{(\mathrm{MSD}, 0.2)}$} & Average & 27.1788 & 10.1794 & 33.1804 & 21.1789 & 42.1812 & 38.1792 \\
\hline & Ave. Abs. Diff $|\mathrm{T}-\mathrm{L}|$ & 0.1956 & 0.1965 & 0.1967 & 0.1951 & 0.1970 & 0.1957 \\
\hline
\end{tabular}

Table 7b. Estimators Ranking based on Tukey HSD Test $\left(n=100, \varepsilon_{1}=0.1, \mu_{1}=10, \varepsilon_{2}=0.2\right)$

\begin{tabular}{cllllllc}
\hline Worst Fit & \multicolumn{1}{c}{} & & & & Best Fit \\
\hline$\overline{\mathbf{X}}$ & $\overline{\mathbf{X}}_{(\mathrm{MSD}, 0.2)}$ & $\overline{\mathbf{X}}_{(\mathrm{MSD}, 0.3)}$ & $\overline{\mathbf{X}}_{(\mathrm{MSD}, 0.4)}$ & $\widehat{\mathbf{M}}$ & $\widehat{\mathbf{M}}_{(\mathrm{MSD}, 0.2)}$ & $\widehat{\mathbf{M}}_{(\mathrm{MSD}, 0.3)}{ }^{*}$ & $\widehat{\mathbf{M}}_{(\mathrm{MSD}, 0.4)}{ }^{*}$ \\
\hline
\end{tabular}

Note: All pairwise Tukey HSD results are significant at 0.05 significant level

except ${ }^{*} \widehat{\mathrm{M}}_{(\mathrm{MSD}, 0.3)}$ and $\widehat{\mathrm{M}}_{(\mathrm{MSD}, 0.4)}$ are insignificant with $p$-value of 0.091 . 


\subsection{Data Contamination at $\varepsilon_{1}=0.1$ and $\varepsilon_{2}=0.4$}

\subsubsection{Mild Cellwise Outliers $\left(\mu_{1}=5\right)$}

With $\varepsilon_{1}$ maintained at 0.1 and $\varepsilon_{2}$ increased from 0.2 to 0.4 , the total outlying proportion in the data added up to $50 \%$. The $\overline{\mathbf{X}}$ estimation is now severely deviated from $L_{\mathrm{p}}$ regardless of sample sizes (Tables 8a and 9a). Obvious deviation also can be observed in $\overline{\mathbf{X}}_{(\mathbf{M S D}, \boldsymbol{\alpha})}$ when the outlying proportion is greater than trimming percentages $(\alpha$ $=0.2,0.3,0.4$ ), while $\widehat{\mathbf{M}}$ starts showing increase in deviation, as well. Meanwhile, noticeable differences in $\widehat{\mathbf{M}}_{(\mathbf{M S D}, \boldsymbol{\alpha})}$ between the trimming percentages can be detected, as higher $\alpha$ will have lower $|\mathrm{T}-\mathrm{L}|$.

The ANOVA test and Tukey HSD test for $n=40$ $(p=0.000)$ and $n=100(p=0.000)$ have reported significant difference in performance between the estimators. Tables $8 \mathrm{~b}$ and $9 \mathrm{~b}$ show identical rankings for estimators, with $\widehat{\mathbf{M}}_{(\mathbf{M S D}, \mathbf{0 . 4})}$ has the best fit while $\overline{\mathbf{X}}$ has the least fit for both sample size $n=40$ and $n=100$.

\subsubsection{Severe Cellwise Outliers $\left(\mu_{1}=10\right)$}

The most severe outlying condition in the study, $\varepsilon_{1}=0.1$, $\mu_{1}=10$ and $\varepsilon_{2}=0.4$, was simulated and reported into Table 10a and Table 11a for $n=40$ and $n=100$ respectively.

As expected, $\overline{\mathrm{X}}$ deviates the most from $L_{\mathrm{p}}$ with average deviation of 6.997 for both $n=40$ and $n=100$ under each dimension. Further deviation also can be seen on $\overline{\mathrm{X}}_{(\mathrm{MSD}, \alpha)}$, which corresponds with the cell wise outliers' inflation.

However, $\widehat{\mathbf{M}}$ performance maintains at this level of outliers' proportions, $\varepsilon_{1}=0.1$ and $\varepsilon_{2}=0.4$, despite the cellwise outliers' inflation. Similarly, $\widehat{\mathbf{M}}_{(\mathbf{M S D}, \boldsymbol{\alpha})}$ 's deviation does not affected much from the inflation, maintaining its low deviation even at higher trimming percentage.

Lastly, ANOVA test again indicates significant difference on estimators' performance with $p=0.000$ for $n$ $=40$ and $n=100$. The ranking of the estimators is similar to the previous section (as shown in Tables 10b and 11b).

Table 8a. Simulation Parameter Settings ( $\left.n=40, \varepsilon_{1}=0.1, \mu_{1}=5, \varepsilon_{2}=0.4\right)$

\begin{tabular}{|c|c|c|c|c|c|c|c|}
\hline \multicolumn{2}{|c|}{ Location Estimators (T) } & \multicolumn{6}{|c|}{ Pre-set Location $\left(L_{p}\right)$} \\
\hline & & 27 & 10 & 33 & 21 & 42 & 38 \\
\hline \multirow{2}{*}{$\overline{\mathbf{X}}$} & Average & 33.4903 & 16.4767 & 39.4676 & 27.5324 & 48.4886 & 44.5046 \\
\hline & Ave. Abs. Diff $|\mathrm{T}-\mathrm{L}|$ & 6.4974 & 6.4820 & 6.4746 & 6.5406 & 6.4971 & 6.5120 \\
\hline \multirow{2}{*}{$\widehat{\mathbf{M}}$} & Average & 27.7786 & 10.7759 & 33.7732 & 21.7850 & 42.7767 & 38.7792 \\
\hline & Ave. Abs. Diff $|\mathrm{T}-\mathrm{L}|$ & 0.7833 & 0.7820 & 0.7802 & 0.7912 & 0.7846 & 0.7855 \\
\hline \multirow{2}{*}{$\overline{\mathbf{X}}_{(\mathbf{M S D}, \mathbf{0 . 4})}$} & Average & 27.8379 & 10.8354 & 33.8372 & 21.8405 & 42.8360 & 38.8402 \\
\hline & Ave. Abs. Diff $|\mathrm{T}-\mathrm{L}|$ & 0.8380 & 0.8354 & 0.8372 & 0.8405 & 0.8360 & 0.8402 \\
\hline \multirow{2}{*}{$\overline{\mathbf{X}}_{(\mathbf{M S D}, 0.3)}$} & Average & 29.4462 & 12.4359 & 35.4263 & 23.4202 & 44.4207 & 40.4206 \\
\hline & Ave. Abs. Diff $|\mathrm{T}-\mathrm{L}|$ & 2.4840 & 2.4783 & 2.4743 & 2.4666 & 2.4624 & 2.4609 \\
\hline \multirow{2}{*}{$\overline{\mathbf{X}}_{(\mathbf{M S D}, 0.2)}$} & Average & 30.9615 & 13.9400 & 36.9208 & 24.9491 & 45.9403 & 41.9223 \\
\hline & Ave. Abs. Diff $|\mathrm{T}-\mathrm{L}|$ & 3.9977 & 3.9781 & 3.9652 & 3.9908 & 3.9777 & 3.9537 \\
\hline \multirow{2}{*}{$\widehat{\mathbf{M}}_{(\mathbf{M S D}, 0.4)}$} & Average & 27.2522 & 10.2499 & 33.2518 & 21.2533 & 42.2475 & 38.2556 \\
\hline & Ave. Abs. Diff $|\mathrm{T}-\mathrm{L}|$ & 0.3062 & 0.3014 & 0.3053 & 0.3058 & 0.3041 & 0.3084 \\
\hline \multirow{2}{*}{$\widehat{\mathbf{M}}_{(\mathbf{M S D}, 0.3)}$} & Average & 27.3795 & 10.3763 & 33.3789 & 21.3807 & 42.3746 & 38.3832 \\
\hline & Ave. Abs. Diff $|\mathrm{T}-\mathrm{L}|$ & 0.4084 & 0.4056 & 0.4086 & 0.4108 & 0.4070 & 0.4131 \\
\hline \multirow{2}{*}{$\widehat{\mathbf{M}}_{(\mathrm{MSD}, 0.2)}$} & Average & 27.5086 & 10.5052 & 33.5054 & 21.5101 & 42.5034 & 38.5077 \\
\hline & Ave. Abs. Diff $|\mathrm{T}-\mathrm{L}|$ & 0.5246 & 0.5230 & 0.5240 & 0.5290 & 0.5221 & 0.5258 \\
\hline
\end{tabular}

Table 8b. Estimators Ranking based on Tukey HSD Test $\left(n=40, \varepsilon_{1}=0.1, \mu_{1}=5, \varepsilon_{2}=0.4\right)$

\begin{tabular}{cccccccc}
\hline Worst Fit & & & & & & Best Fit \\
\hline$\overline{\mathbf{X}}$ & $\overline{\mathbf{X}}_{(\mathrm{MSD}, 0.2)}$ & $\overline{\mathbf{X}}_{(\mathrm{MSD}, 0.3)}$ & $\overline{\mathbf{X}}_{(\mathrm{MSD}, 0.4)}$ & $\widehat{\mathbf{M}}$ & $\widehat{\mathbf{M}}_{(\mathrm{MSD}, 0.2)}$ & $\widehat{\mathbf{M}}_{(\mathrm{MSD}, 0.3)}$ & $\widehat{\mathbf{M}}_{(\mathrm{MSD}, 0.4)}$ \\
\hline
\end{tabular}

Note: All pairwise Tukey HSD results are significant at 0.05 significant level 
Table 9a. Simulation Parameter Settings ( $\left.n=100, \varepsilon_{1}=0.1, \mu_{1}=5, \varepsilon_{2}=0.4\right)$

\begin{tabular}{|c|c|c|c|c|c|c|c|}
\hline \multirow{2}{*}{\multicolumn{2}{|c|}{ Location Estimators (T) }} & \multicolumn{6}{|c|}{ Pre-set Location $\left(L_{\mathrm{p}}\right)$} \\
\hline & & 27 & 10 & 33 & 21 & 42 & 38 \\
\hline \multirow{2}{*}{$\overline{\mathbf{x}}$} & Average & 33.5093 & 16.5053 & 39.4953 & 27.4935 & 48.4974 & 44.4803 \\
\hline & Ave. Abs. Diff $|\mathrm{T}-\mathrm{L}|$ & 6.5093 & 6.5053 & 6.4953 & 6.4935 & 6.4975 & 6.4803 \\
\hline \multirow{2}{*}{$\widehat{\mathbf{M}}$} & Average & 27.7574 & 10.7575 & 33.7545 & 21.7554 & 42.7574 & 38.7558 \\
\hline & Ave. Abs. Diff |T-L| & 0.7575 & 0.7577 & 0.7547 & 0.7555 & 0.7576 & 0.7559 \\
\hline \multirow{2}{*}{$\overline{\mathbf{X}}_{(\mathrm{MSD}, 0.4)}$} & Average & 27.8335 & 10.8350 & 33.8352 & 21.8345 & 42.8362 & 38.8347 \\
\hline & Ave. Abs. Diff $|\mathrm{T}-\mathrm{L}|$ & 0.8335 & 0.8350 & 0.8352 & 0.8345 & 0.8362 & 0.8347 \\
\hline \multirow{2}{*}{$\overline{\mathbf{X}}_{(\mathrm{MSD}, 0.3)}$} & Average & 29.3949 & 12.3975 & 35.3905 & 23.3757 & 44.3889 & 40.3816 \\
\hline & Ave. Abs. Diff $|\mathrm{T}-\mathrm{L}|$ & 2.3963 & 2.3988 & 2.3913 & 2.3766 & 2.3899 & 2.3830 \\
\hline \multirow{2}{*}{$\overline{\mathbf{X}}_{(\mathrm{MSD}, 0.2)}$} & Average & 30.8926 & 13.8929 & 36.8797 & 24.8796 & 45.8869 & 41.8764 \\
\hline & Ave. Abs. Diff $|\mathrm{T}-\mathrm{L}|$ & 3.8939 & 3.8936 & 3.8810 & 3.8803 & 3.8872 & 3.8771 \\
\hline \multirow{2}{*}{$\widehat{\mathbf{M}}_{(\mathbf{M S D}, \mathbf{0 . 4})}$} & Average & 27.2514 & 10.2519 & 33.2530 & 21.2537 & 42.2513 & 38.2531 \\
\hline & Ave. Abs. Diff $|\mathrm{T}-\mathrm{L}|$ & 0.2637 & 0.2637 & 0.2651 & 0.2653 & 0.2640 & 0.2651 \\
\hline \multirow{2}{*}{$\widehat{\mathbf{M}}_{(\mathbf{M S D}, 0.3)}$} & Average & 27.3815 & 10.3822 & 33.3817 & 21.3812 & 42.3803 & 38.3808 \\
\hline & Ave. Abs. Diff $|\mathrm{T}-\mathrm{L}|$ & 0.3850 & 0.3855 & 0.3851 & 0.3841 & 0.3841 & 0.3843 \\
\hline \multirow{2}{*}{$\widehat{\mathbf{M}}_{(\mathrm{MSD}, 0.2)}$} & Average & 27.5069 & 10.5078 & 33.5059 & 21.5078 & 42.5077 & 38.5082 \\
\hline & Ave. Abs. Diff $|\mathrm{T}-\mathrm{L}|$ & 0.5081 & 0.5089 & 0.5074 & 0.5087 & 0.5090 & 0.5092 \\
\hline
\end{tabular}

Table 9b. Estimators Ranking based on Tukey HSD Test $\left(n=100, \varepsilon_{1}=0.1, \mu_{1}=5, \varepsilon_{2}=0.4\right)$

\begin{tabular}{clllllll}
\hline Worst Fit & & & & & & Best Fit \\
\hline$\overline{\mathbf{X}}$ & $\overline{\mathbf{X}}_{(\mathrm{MSD}, 0.2)}$ & $\overline{\mathbf{X}}_{(\mathrm{MSD}, 0.3)}$ & $\overline{\mathbf{X}}_{(\mathrm{MSD}, \mathbf{0 . 4})}$ & $\widehat{\mathbf{M}}$ & $\widehat{\mathbf{M}}_{(\mathrm{MSD}, 0.2)}$ & $\widehat{\mathbf{M}}_{(\mathrm{MSD}, 0.3)}$ & $\widehat{\mathbf{M}}_{(\mathrm{MSD}, 0.4)}$ \\
\hline
\end{tabular}

Note: All pairwise Tukey HSD results are significant at 0.05 significant level

Table 10a. Simulation Parameter Settings $\left(n=40, \varepsilon_{1}=0.1, \mu_{1}=10, \varepsilon_{2}=0.4\right)$

\begin{tabular}{|c|c|c|c|c|c|c|c|}
\hline \multirow{2}{*}{\multicolumn{2}{|c|}{ Location Estimators (T) }} & \multicolumn{6}{|c|}{ Pre-set Location $\left(L_{\mathrm{p}}\right)$} \\
\hline & & 27 & 10 & 33 & 21 & 42 & 38 \\
\hline \multirow{2}{*}{$\overline{\mathbf{X}}$} & Average & 33.9903 & 16.9767 & 39.9676 & 28.0324 & 48.9886 & 45.0046 \\
\hline & Ave. Abs. Diff $|\mathrm{T}-\mathrm{L}|$ & 6.9937 & 6.9791 & 6.9717 & 7.0367 & 6.9934 & 7.0085 \\
\hline \multirow{2}{*}{$\widehat{\mathbf{M}}$} & Average & 27.7774 & 10.7757 & 33.7724 & 21.7897 & 42.7799 & 38.7793 \\
\hline & Ave. Abs. Diff $|\mathrm{T}-\mathrm{L}|$ & 0.7832 & 0.7820 & 0.7791 & 0.7964 & 0.7873 & 0.7851 \\
\hline \multirow{2}{*}{$\overline{\mathbf{X}}_{(\mathbf{M S D}, \mathbf{0 . 4})}$} & Average & 28.6833 & 11.6855 & 34.6843 & 22.6871 & 43.6837 & 39.6884 \\
\hline & Ave. Abs. Diff $|\mathrm{T}-\mathrm{L}|$ & 1.6833 & 1.6855 & 1.6843 & 1.6871 & 1.6837 & 1.6884 \\
\hline \multirow{2}{*}{$\overline{\mathbf{X}}_{(\mathrm{MSD}, 0.3)}$} & Average & 30.1593 & 13.1527 & 36.1350 & 24.1264 & 45.1316 & 41.1317 \\
\hline & Ave. Abs. Diff $|\mathrm{T}-\mathrm{L}|$ & 3.1674 & 3.1611 & 3.1467 & 3.1389 & 3.1417 & 3.1419 \\
\hline \multirow{2}{*}{$\overline{\mathbf{X}}_{(\mathbf{M S D}, 0.2)}$} & Average & 31.5740 & 14.5487 & 37.5371 & 25.5597 & 46.5563 & 42.5282 \\
\hline & Ave. Abs. Diff $|\mathrm{T}-\mathrm{L}|$ & 4.5904 & 4.5654 & 4.5569 & 4.5769 & 4.5718 & 4.5409 \\
\hline \multirow{2}{*}{$\widehat{\mathbf{M}}_{(\mathbf{M S D}, 0.4)}$} & Average & 27.2517 & 10.2508 & 33.2535 & 21.2574 & 42.2522 & 38.2558 \\
\hline & Ave. Abs. Diff $|\mathrm{T}-\mathrm{L}|$ & 0.3058 & 0.3031 & 0.3056 & 0.3094 & 0.3069 & 0.3082 \\
\hline \multirow{2}{*}{$\widehat{\mathbf{M}}_{(\mathbf{M S D}, 0.3)}$} & Average & 27.3775 & 10.3760 & 33.3787 & 21.3820 & 42.3756 & 38.3814 \\
\hline & Ave. Abs. Diff $|\mathrm{T}-\mathrm{L}|$ & 0.4069 & 0.4059 & 0.4081 & 0.4117 & 0.4075 & 0.4103 \\
\hline \multirow{2}{*}{$\widehat{\mathbf{M}}_{(\mathbf{M S D}, 0.2)}$} & Average & 27.5069 & 10.5037 & 33.5051 & 21.5097 & 42.5049 & 38.5059 \\
\hline & Ave. Abs. Diff $|\mathrm{T}-\mathrm{L}|$ & 0.5245 & 0.5217 & 0.5235 & 0.5281 & 0.5232 & 0.5232 \\
\hline
\end{tabular}

Table 10b. Estimators Ranking based on Tukey HSD Test $\left(n=40, \varepsilon_{1}=0.1, \mu_{1}=10, \varepsilon_{2}=0.4\right)$

\begin{tabular}{|c|c|c|c|c|c|c|c|}
\hline Worst Fit & & & & & & & Best Fit \\
\hline$\overline{\mathbf{X}}$ & $\overline{\mathbf{X}}_{(\mathbf{M S D}, 0.2)}$ & $\overline{\mathbf{X}}_{(\mathrm{MSD}, 0.3)}$ & $\overline{\mathbf{X}}_{(\mathbf{M S D}, \mathbf{0 . 4})}$ & $\widehat{\mathbf{M}}$ & $\widehat{\mathbf{M}}_{(\mathrm{MSD}, 0.2)}$ & $\widehat{\mathbf{M}}_{(\mathbf{M S D}, 0.3)}$ & $\widehat{\mathbf{M}}_{(\mathbf{M S D}, 0.4)}$ \\
\hline
\end{tabular}

Note: All pairwise Tukey HSD results are significant at 0.05 significant level 
Table 11a. Simulation Parameter Settings ( $\left.n=100, \varepsilon_{1}=0.1, \mu_{1}=10, \varepsilon_{2}=0.4\right)$

\begin{tabular}{|c|c|c|c|c|c|c|c|}
\hline \multirow{2}{*}{\multicolumn{2}{|c|}{ Location Estimators (T) }} & \multicolumn{6}{|c|}{ Pre-set Location $\left(L_{\mathrm{p}}\right)$} \\
\hline & & 27 & 10 & 33 & 21 & 42 & 38 \\
\hline \multirow{2}{*}{$\overline{\mathbf{X}}$} & Average & 34.0093 & 17.0053 & 39.9953 & 27.9935 & 48.9974 & 44.9803 \\
\hline & Ave. Abs. Diff $|\mathrm{T}-\mathrm{L}|$ & 7.0093 & 7.0053 & 6.9953 & 6.9935 & 6.9974 & 6.9803 \\
\hline \multirow{2}{*}{$\widehat{\mathbf{M}}$} & Average & 27.7555 & 10.7586 & 33.7544 & 21.7555 & 42.7595 & 38.7546 \\
\hline & Ave. Abs. Diff $|\mathrm{T}-\mathrm{L}|$ & 0.7556 & 0.7587 & 0.7546 & 0.7556 & 0.7597 & 0.7547 \\
\hline \multirow{2}{*}{$\overline{\mathbf{X}}_{(\text {MSD, }, 0.4)}$} & Average & 28.6711 & 11.6739 & 34.6732 & 22.6734 & 43.6744 & 39.6718 \\
\hline & Ave. Abs. Diff |T-L| & 1.6711 & 1.6739 & 1.6732 & 1.6734 & 1.6744 & 1.6718 \\
\hline \multirow{2}{*}{$\overline{\mathbf{X}}_{(\mathrm{MSD}, 0.3)}$} & Average & 30.1152 & 13.1161 & 36.1091 & 24.0942 & 45.1065 & 41.1022 \\
\hline & Ave. Abs. Diff $|\mathrm{T}-\mathrm{L}|$ & 3.1153 & 3.1163 & 3.1091 & 3.0942 & 3.1066 & 3.1023 \\
\hline \multirow{2}{*}{$\overline{\mathbf{X}}_{(\mathrm{MSD}, 0.2)}$} & Average & 31.5109 & 14.5127 & 37.4982 & 25.5011 & 46.5064 & 42.4971 \\
\hline & Ave. Abs. Diff |T-L| & 4.5113 & 4.5127 & 4.4983 & 4.5014 & 4.5064 & 4.4971 \\
\hline \multirow{2}{*}{$\widehat{\mathbf{M}}_{(\mathbf{M S D}, 0.4)}$} & Average & 27.2502 & 10.2539 & 33.2529 & 21.2541 & 42.2532 & 38.2535 \\
\hline & Ave. Abs. Diff $|\mathrm{T}-\mathrm{L}|$ & 0.2627 & 0.2644 & 0.2653 & 0.2658 & 0.2653 & 0.2657 \\
\hline \multirow{2}{*}{$\widehat{\mathbf{M}}_{(\mathbf{M S D}, 0.3)}$} & Average & 27.3801 & 10.3837 & 33.3808 & 21.3819 & 42.3828 & 38.3818 \\
\hline & Ave. Abs. Diff $|\mathrm{T}-\mathrm{L}|$ & 0.3838 & 0.3864 & 0.3849 & 0.3850 & 0.3867 & 0.3853 \\
\hline \multirow{2}{*}{$\widehat{\mathbf{M}}_{(\mathbf{M S D}, 0.2)}$} & Average & 27.5042 & 10.5085 & 33.5054 & 21.5082 & 42.5086 & 38.5079 \\
\hline & Ave. Abs. Diff $|\mathrm{T}-\mathrm{L}|$ & 0.5054 & 0.5094 & 0.5071 & 0.5093 & 0.5100 & 0.5090 \\
\hline
\end{tabular}

Table 11b. Estimators Ranking based on Tukey HSD Test $\left(n=100, \varepsilon_{1}=0.1, \mu_{1}=10, \varepsilon_{2}=0.4\right)$

\begin{tabular}{cccccccc}
\hline \multicolumn{1}{c}{ Worst Fit } & & & & & & Best Fit \\
\hline$\overline{\mathbf{X}}$ & $\overline{\mathbf{X}}_{(\mathrm{MSD}, 0.2)}$ & $\overline{\mathbf{X}}_{(\mathrm{MSD}, 0.3)}$ & $\overline{\mathbf{X}}_{(\mathrm{MSD}, 0.4)}$ & $\widehat{\mathbf{M}}$ & $\widehat{\mathbf{M}}_{(\mathrm{MSD}, 0.2)}$ & $\widehat{\mathbf{M}}_{(\mathrm{MSD}, 0.3)}$ & $\widehat{\mathbf{M}}_{(\mathrm{MSD}, 0.4)}$ \\
\hline
\end{tabular}

Note: All pairwise Tukey HSD results are significant at 0.05 significant level

\subsection{Summary}

To summarize, the classical mean $(\overline{\mathbf{X}})$ is good when applied to clean data, but very susceptible to the existence of outliers. The performance of classical median $(\widehat{\mathbf{M}})$ is considered moderate as the rankings are consistently in between the other estimators. On the other hand, despite having comparable performance to $\widehat{\mathbf{M}}$ when applied to clean data, $\overline{\mathbf{X}}_{(\mathbf{M S D}, \boldsymbol{\alpha})}$ 's performance worsens when dealing with contaminated data. The estimation through $\overline{\mathbf{X}}_{(\mathbf{M S D}, \boldsymbol{\alpha})}$ increasingly deviates from $L_{\mathrm{p}}$ as the proportion $\left(\varepsilon_{2}\right)$ of casewise outliers increases. The deviation also increases when the cellwise outliers' inflation $\left(\mu_{1}\right)$ increases. However, $\widehat{\mathbf{M}}_{(\mathbf{M S D}, \boldsymbol{\alpha})}$ holds a dominant performance over $\overline{\mathbf{X}}$, $\widehat{\mathbf{M}}$ and $\overline{\mathbf{X}}_{(\mathbf{M S D}, \boldsymbol{\alpha})}$ on all contaminated occasions regardless of the trimming percentage applied. Overall, $\widehat{\mathbf{M}}_{(\mathbf{M S D}, \mathbf{0 . 4})}$ yields best location estimations than the other two trimming percentages $(\alpha=0.2,0.3)$.

\section{Conclusions}

In this paper, the capability of the existing multivariate location estimators in handling situation where both cellwise outliers and casewise outliers coexist is discussed and reviewed. One should take note that when both casewise and cellwise outliers coexist in the dataset, they should be treated separately. Treating the coexistence of these outliers like a single problem could still jeopardize the location measure estimation as the outliers might not be well identified (leftover outliers). Based on the results of simulation study, the proposed distance-based trimmed median, $\widehat{\mathbf{M}}_{(\mathbf{M S D}, \boldsymbol{\alpha})}$, yields more accurate location estimations despite the leftover outliers compared to the other three chosen estimators in this study.

Pertaining the feasibility of using median over mean after $\alpha$-distance-based trimming, the simulation study shows that $\widehat{\mathbf{M}}_{(\mathbf{M S D}, \boldsymbol{\alpha})}$ has consistently lower deviation as compared to $\overline{\mathbf{X}}_{(\mathbf{M S D}, \boldsymbol{\alpha})}$. Despite the performance of both $\widehat{\mathbf{M}}_{(\mathbf{M S D}, \boldsymbol{\alpha})}$ and $\overline{\mathbf{X}}_{(\mathbf{M S D}, \boldsymbol{\alpha})}$ getting better as the trimming percentage $(\alpha)$ increases, in cases where $\alpha$ relatively lower than the total outlying proportions in the data, $\widehat{\mathbf{M}}_{(\mathbf{M S D}, \boldsymbol{\alpha})}$ outperforms $\overline{\mathbf{X}}_{(\mathbf{M S D}, \boldsymbol{\alpha})}$ with noticeable differences. When the contaminated data is under-trimmed, the leftover cellwise and casewise outliers can cause $\overline{\mathbf{X}}_{(\mathbf{M S D}, \boldsymbol{\alpha})}$ to breakdown. On the other hand, even though trimming percentage equal or greater than the total outlying 
proportions in the data, some cellwise outliers might be left from trimming due to the cellwise outliers which are randomly spread within the data. Thus, the use of median after $\alpha$-distance-based trimming is recommended to mitigate the problem.

As a conclusion, the proposed two-stage outlier's filtration location estimator, $\widehat{\mathbf{M}}_{(\mathbf{M S D}, \boldsymbol{\alpha})}$, is recommended as a better multivariate location estimator when dealing with unknown outlier's situation.

\section{Acknowledgements}

This research was supported by Ministry of Higher Education (MoHE) of Malaysia through Fundamental Research Grant Scheme (FRGS/1/2019/STG06/UUM/02/8).

\section{REFERENCES}

[1] F. R. Hampel, "Robust Statistics: A Brief Introduction and Overview”, In Seminar für Statistik, Eidgenössische Technische Hochschule, Switzerland, vol. 94, 2001, pp. 1-5.

[2] F. R. Hampel, E. M. Ronchetti, P. J. Rousseeuw and W. A. Stahel, Robust statistics: the approach based on influence functions, Vol. 196, John Wiley \& Sons, 2011.

[3] R. J. Pell, "Multiple outlier detection for multivariate calibration using robust statistical techniques", Chemometrics and Intelligent Laboratory Systems, vol. 52, no. 1 , 2000, pp. 87-104.

[4] J. Á. Víšek, "Regression with high breakdown point”, in Proceedings of ROBUST, Sept.2000, pp. 324-356.

[5] F. R. Hampel, "The breakdown points of the mean combined with some rejection rules”, Technometrics, vol 27, no. 2, 1985, pp. 95-107.

[6] P. J. Rousseeuw, "Multivariate estimation with high breakdown point”, Mathematical statistics and applications, vol. 8, 1985, pp. 283-297.

[7] D. M. Hawkins, “Outliers”, in Encyclopedia of Biostatistics, P. Armitage and T. Colton, Eds. John Wiley \& Sons, 2005, pp. 4233-4237.

[8] I. M. Rodrigues and G. Boente, "Multivariate Outliers”, in International Encyclopedia of Statistical Science , M. Lovric, Ed. Springer-Verlag Berlin Heidelberg, 2011, pp. 910-912.

[9] C. Agostinelli, A. Leung, V. J. Yohai and R. H. Zamar, "Robust estimation of multivariate location and scatter in the presence of cellwise and casewise contamination”, TEST, vol. 24, no. 3, 2015, pp. 441-461.

[10] P. J. Rousseeuw and W. Van den Bossche, "Comments on: Robust estimation of multivariate location and scatter in the presence of cellwise and casewise contamination", TEST, vol. 24, no. 3, 2015, pp. 473-477.

[11] J. W. Tukey, "Some elementary problems of importance to small sample practice”, Human Biology, vol. 20, no. 4, 1948, pp. 205-214.

[12] D. K. Srivastava and G. S. Mudholkar, "Trimmed T2: A robust analog of hotelling's T2”, Journal of Statistical Planning and Inference, vol. 97, no. 2, 2001, pp. 343-358.

[13] J. Alloway and M. Raghavachari, "Multivariate control charts based on trimmed means", in ASQC Quality Congress Transactions, San Francisco, 1990, pp. 449-453.

[14] Y. S. Pang, N. A. Ahad, S. S. Syed Yahaya and Y. F. Lim, "Robust Linear Discriminant Rule Using Novel Distance-based Trimming Procedure", Jour of Adv Research in Dynamical \& Control Systems, vol. 11, 05-Special Issue, 2019, pp. 969-978.

[15] G. McLachlan, "Mahalanobis distance", Resonance, vol. 4, no. 6, 1999, pp. 20-26.

[16] D. M. Rocke, G. W. Downs and A. J. Rocke, “Are robust estimators really necessary?”, Technometrics, vol. 24, no. 2, 1982, pp. 95-101.

[17] M. Wu, “Trimmed and winsorized estimators," Ph.D. dissertation, Prob. And Stat. Dept., Michigan State Univ., East Lansing, MI, 2007.

[18] R. R. Wilcox, “Trimming and Winsorization”, in Encyclopedia of Biostatistics, New York: John Wiley \& Sons, Inc, 2005.

[19] J. L. Rosenberger and M. Gasko, "Comparing location estimators: Trimmed means, medians, and trimean", Understanding robust and exploratory data analysis, 1983, pp. 297-336.

[20] A. R. Othman, H. J. Keselman, A. R. Padmanabhan, R. R. Wilcox and K. Fradette, "Comparing measures of the 'typical' score across treatment groups”, British Journal of Mathematical and Statistical Psychology, vol. 57, no. 2, 2004, pp. 215-234.

[21] H. J. Keselman, A. R. Othman, R. R. Wilcox and K. Fradette, "The new and improved two-sample t test", Psychological Science, vol. 15, no. 1, 2004, pp. 47-51.

[22] P. J. Huber, "Robust Estimation of a Location Parameter", The Annals of Mathematical Statistics, vol. 35, no. 1, 1964, pp. 73-101.

[23] J. W. Tukey, "The future of data analysis”, The annals of mathematical statistics, vol. 33, no. 1, 1962, pp. 1-67. 